\title{
Cardiopulmonary protection of modified remote ischemic preconditioning in mitral valve replacement surgery: A randomized controlled trial
}

Kang Zhou

Xuzhou medical university https://orcid.org/0000-0002-7237-2247

Lianqin Zhang

The second affiliated hospital of soochow university

Tianzhu Gu

Xuzhou Medical University

Jingjing Xu

Xuzhou Medical University

Mengzhu Shi

Xuzhou Medical University

Jiang Zhu

The second affiliated hospital of soochow university

Jindong Liu ( $\square$ liujindong1818@163.com )

The Affiliated Hospital of Xuzhou Medical University

\section{Research article}

Keywords: Remote ischemic preconditioning, Cardiopulmonary function, Myocardial enzymes, Mitral valve replacement, Acute lung injury

Posted Date: January 25th, 2021

DOl: https://doi.org/10.21203/rs.3.rs-152372/v1

License: (a) (1) This work is licensed under a Creative Commons Attribution 4.0 International License. Read Full License 


\section{Abstract}

\section{Background}

Remote ischemic preconditioning (RIPC) is reported to have early-phase and delayed-phase organprotective effects. Whether the modified RIPC (mRIPC) protocol performed repeatedly provides cardiopulmonary protection is still uncertain.

\section{Methods}

In this single-center, randomized, controlled trial, 86 patients undergoing elective mitral valve replacement (MVR) surgery were randomized 1:1 to receive either mRIPC or no ischemic preconditioning (control). Three cycles of $5 \mathrm{~min}$ ischemia and 5 min reperfusion induced by a blood pressure cuff served as the RIPC stimulus. Modified RIPC was induced at the following three time points: $24 \mathrm{~h}, 12 \mathrm{~h}$ and $1 \mathrm{~h}$ before surgery. Blood samples were withdrawn at $10 \mathrm{~min}$ after intubation (T0), at $1 \mathrm{~h}$ after aortic declamping

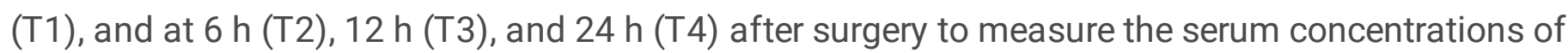
myocardial enzymes and other biomarkers, including cardiac troponin I (cTnl), which was the primary end point of this study. Creatine kinase isoenzyme (CK-MB), lactate dehydrogenase (LDH), inotropic score (IS) and inflammatory mediators were also measured. Blood gas analysis was conducted to calculate the $\mathrm{PaO}_{2} / \mathrm{FiO}_{2}$ ratio and $\mathrm{A}-\mathrm{aDO}_{2}$, and the incidence of acute lung injury (ALI) was also recorded.

\section{Results}

Modified RIPC significantly decreased the serum concentrations of cTnl, CK-MB and LDH at T2, T3 and T4 $(P<0.01)$, and the IS decreased compared with that in the control group $(11.96 \pm 0.92$ vs. $14.20 \pm 1.09$, $P<0.01)$. In addition, the incidence of ALI in the MRIPC group was decreased $(18.60 \%$ vs. $34.88 \%, P=$ $0.046)$, and the $\mathrm{PaO}_{2} / \mathrm{FiO}_{2}$ was higher at $\mathrm{T} 4(P<0.05)$. Compared with those in the control group, the levels of interleukin 6 (IL-6) and tumor necrosis factor-a (TNF-a) were decreased at T1, T2, T3 and T4 ( $P<$ $0.05)$ in the mRIPC group, and the level of IL-10 increased at the same time.

\section{Conclusions}

Modified RIPC decreased the incidence of myocardial and lung injury in MVR surgery, providing new evidence for the clinical application of RIPC in valve surgery.

Trial registration

This study was registered in ClinicalTrials.gov (NCT03010839). Date of Registration on November 5th, 2016.

\section{Background}


Cardiac heart disease is a major cause of death and disability worldwide [1]. Mitral valve injury caused by rheumatic heart disease seriously affects the health and quality of life of patients. At present, mitral valve replacement (MVR) under cardiopulmonary bypass (CPB) is still one of the major treatments for mitral valve disease. However, heart and lung ischemia-reperfusion (I/R) injury during CPB can increase the incidence of postoperative cardiopulmonary complications in patients [2].

Remote ischemic preconditioning (RIPC), induced by brief episodes of blood pressure cuff inflation and deflation on the upper arm or thigh, can help remote vital organs withstand a subsequent prolonged ischemic event [3]. RIPC has been proven to provide cardiopulmonary and renal protection in cardiac surgery $[4,5]$. However, the results of two large multicenter studies in recent years demonstrated that RIPC had no significant effect on clinical outcomes, particularly in patients undergoing coronary artery bypass grafting (CABG) [6, 7]. The following protective effects of RIPC have hitherto been identified: the 'early window of protection', which occurs immediately after the stimulus, lasts $2-3 \mathrm{~h}$ [8], and the 'second window of protection', which is evident $12-24 \mathrm{~h}$ after the stimulus, could be maintained for up to $48-72 \mathrm{~h}$ [9]. The protection of the early phase is short but more effective in terms of increasing the tolerance of myocardial ischemia, while the delayed phase provides this protection more sustained and protects against myocardial stunning.

Most previous studies have focused on CABG surgery, while few studies have focused on cardiopulmonary function injury after valve surgery. Moreover, RIPC is mostly a single intervention, and the optimal stimulation mode (time, intensity) of RIPC is still controversial. In this study, a modified RIPC (mRIPC) method, which was performed at $24 \mathrm{~h}, 12 \mathrm{~h}$ and $1 \mathrm{~h}$ before surgery, was used for intervention. We expect that the delayed phase protection induced by performing mRIPC $24 \mathrm{~h}$ and $12 \mathrm{~h}$ before surgery and the two distinct windows of protection induced by performing mRIPC $1 \mathrm{~h}$ before surgery can overlap to enhance the cardioprotection. We conducted this single-center, randomized, controlled clinical trial to assess whether mRIPC could provide cardiopulmonary protection in patients undergoing MVR surgery.

\section{Methods}

A single-center, prospective, randomized, clinical trial was conducted on patients undergoing elective mitral valve replacement (MVR) surgery. The study was approved by the ethics committee of the Affiliated Hospital of Xuzhou Medical University, Xuzhou, Jiangsu Province, China (XYFY2016-KL035-01). It was performed in compliance with the Declaration of Helsinki. All participants provided written informed consent and this study was registered in ClinicalTrials.gov (NCT03010839). This manuscript adheres to the applicable CONSORT guidelines.

\section{Study participants}

Eligible patients were adults with mitral valve disease who were scheduled to undergo mitral valve replacement surgery under cardiopulmonary bypass (CPB) between November 2017 and August 2019. The exclusion criteria were as follows: urgent surgery, preoperative mechanical ventilator support, systemic infection, severe peripheral vascular disease, drug therapy with sulfonylureas and nicorandil, 
ejection fraction (EF) less than 40\%, severe lung diseases such as chronic obstructive pulmonary disease (COPD), peripheral arterial disease affecting the upper limbs or inclusion in other studies.

\section{Randomization and Allocation}

The patients were randomized in a 1:1 ratio to undergo either modified remote ischemic preconditioning (mRIPC group) or no preconditioning (control group). The randomization sequence was generated by a computer and kept in sealed envelopes. The allocation details were sealed in numbered and opaque envelopes, and each treatment allocation was revealed by an independent staff member opening the envelope before the first mRIPC treatment and was supervised by an independent statistician. Because of the setting of the control group, the patients and operators were informed of the intervention measures, but the statistician did not know the actual situation.

\section{Intervention: Modified RIPC Protocol}

Modified RIPC was induced repeatedly at $24 \mathrm{~h}, 12 \mathrm{~h}$ and $1 \mathrm{~h}$ before surgery to reinforce the protective effects of RIPC. The single RIPC protocol entailed three cycles of upper limb ischemia. A standard blood pressure cuff was placed on the right upper arm, and then the cuff was inflated to $200 \mathrm{~mm} \mathrm{Hg}$ for $5 \mathrm{~min}$, followed by 5 min of cuff deflation [10]. The control group had no interventions. The details of the mRIPC protocol are provided in Figure 2.

\section{Anesthetic and Surgical Management}

Anesthesia was induced with intravenous etomidate $(0.2 \mathrm{mg} / \mathrm{kg})$, sufentanil $(1 \mu \mathrm{g} / \mathrm{kg})$ and rocuronium bromide $(0.6 \mathrm{mg} / \mathrm{kg})$. All patients received continuous perioperative monitoring, including electrocardiography, pulse oximetry, radial arterial blood pressure (ABP), central venous pressure (CVP), rectal temperature, entropy index and transesophageal echocardiography (TEE). After intubation, the patients were mechanically ventilated with a tidal volume of $6-8 \mathrm{ml} / \mathrm{kg}$, an inspiratory to expiratory time ratio of $1: 1.5$, and a fractional inspired oxygen of $60 \%$, and respiratory rates were adjusted to maintain arterial carbon dioxide between 35 and 45 mmHg (Primus1 ventilator; Dräger Medical, Germany). Anesthesia was maintained with propofol $(1.5 \mu \mathrm{g} / \mathrm{ml})$ by target-controlled infusion $(\mathrm{TCl})$.

All patients underwent nonpulsatile CPB and received standard management. Heparin $(2.5 \mathrm{mg} / \mathrm{kg})$ was administered after sternotomy to achieve an active clotting time (ACT) longer than $480 \mathrm{~s}$, a whole blood cardioplegia was perfused to the aortic root after aortic cross-clamping for cardiac arrest, standard cardioplegia was given at $4{ }^{\circ} \mathrm{C}$ with a $4: 1$ blood mixture administered in antegrade fashion $(15-20 \mathrm{ml} / \mathrm{kg})$ for a total volume of approximately $1 \mathrm{~L}$, redosing was redosed at 30-minute intervals. The mean ABP was maintained at 60-70 mmHg, and blood glucose levels were maintained at lower than $<200 \mathrm{mg} / \mathrm{dl}$ during CPB. After aorta declamping, anesthesiologist conducted the TEE examination and use the different vasoactive drugs to maintain a stable circulation. After weaning from CPB, protamine was administered for the reversal of heparin, and the ratio of protamine to heparin was 1.0-1.5. All patients were transferred to the intensive care unit (ICU) and received standardized routine postoperative management. 


\section{Outcomes}

The primary endpoint of this study was the concentrations of cardiac troponin I (cTnl), creatine kinase isoenzyme (CK-MB) and lactate dehydrogenase (LDH) in serum, which were also used to measure myocardial function. Secondary endpoints included the inotropic score (IS), the rate of spontaneous return to cardiac rhythm and the incidence of reperfusion arrhythmia. The spontaneous return to cardiac rhythm was defined as the spontaneous recovery of the heartbeat without external stimulation after cardiopulmonary bypass, reperfusion arrhythmias included the following types: serious bradycardia(HR $<40$ beats / $\mathrm{min}$ ), atrial fibrillation, frequent ventricular premature beats, ventricular fibrillation. The inotropic score (IS) was calculated with the following formula: (dopamine + dobutamine $\times 1)+$ (milrinone $\times 15)+($ epinephrine + norepinephrine + isoproterenol $\times 100)$. The evaluation indexes of postoperative pulmonary function included the $\mathrm{PaO}_{2} / \mathrm{FiO}_{2}$ ratio and alveolar-arterial oxygen gradient $\left(\mathrm{A}-\mathrm{aDO}_{2}\right)$ according to the results of arterial blood gas analysis. The incidence of acute lung injury (ALI) was also assessed. Acute lung injury (ALI) was defined as impaired oxygenation with a $\mathrm{PaO} 2 / \mathrm{FiO} 2$ ratio of $<300$ $\mathrm{mm} \mathrm{Hg}$, excluding bronchopneumonia, aspiration, cardiac origin pulmonary edema and fluid overload. In addition, some inflammatory mediators, including interleukin-6 (IL-6), interleukin-10 (IL-10) and tumor necrosis factor- $a$ (TNF-a), were also included. Lactic acid (Lac), as an indicator of metabolism, was also measured. All blood samples were collected at $10 \mathrm{~min}$ after intubation (T0), at $1 \mathrm{~h}$ after aortic declamping (T1), and at $6 \mathrm{~h}$ (T2), $12 \mathrm{~h}$ (T3) and $24 \mathrm{~h}$ (T4) after the completion of surgery. The ventilation time, intensive care unit (ICU) stay time and length of stay (LOS) data were collected.

\section{Blood samples}

The blood samples were collected and centrifuged immediately at $3,000 \mathrm{rpm}$ for $10 \mathrm{~min}$ at $4^{\circ} \mathrm{C}$, and then serum was frozen at $-80^{\circ} \mathrm{C}$ for later analysis by enzyme-linked immunosorbent assay (ELISA) according to Engvall and Perlmann [11] with a commercially available kit (Wuhan Biofavor Biotech Services Co., Wuhan, China). Arterial blood samples were also collected for the blood gas analyses to calculate the $\mathrm{PaO}_{2} / \mathrm{FiO}_{2}$ ratio and $\mathrm{A}-\mathrm{aDO}_{2}$ using a blood gas system (Roche Diagnostics $\mathrm{GmbH}$, Germany).

\section{Statistical analysis}

According to the results of the pilot study, the data with the minimum difference in cTnl concentration between the two groups at several time points after surgery were used to calculate the sample size. In this situation, the value in the mRIPC group was $3.11 \pm 0.49$, and the value in the control group was $3.50 \pm$ 0.51 . At least 36 patients were required per group when simulating the probability of a Type I error (a) at 0.05 and a Type II error at 0.1 ( $\beta$ ). Considering a $15 \%$ dropout rate, the sample size was set at 43 patients per group.

Continuous data were analyzed with the Shapiro-Wilk test to determine whether they conformed to a normal distribution. Measurement data that obeyed a normal distribution are expressed as the mean \pm standard deviation $(\mathrm{X} \pm \mathrm{SD})$, measurement data with a non-normal distribution are expressed as the median (interquartile range, IQR), and categorical variables are expressed as percentages. T-test was used 
to compare two groups of measurement data with normal distributions, the Mann-Whitney U test was used for measurement data with non-normal distributions, and the chi-square test or Fisher's exact probability method was used for categorical data. Repeated measurement data at multiple time points were analyzed by repeated measurement analysis of variance (ANOVA). A $P$ value less than 0.05 was considered significant. All statistical analyses were performed with SPSS statistical software, version 16.0 (SPSS Inc., Chicago, IL).

\section{Results}

In this study, 415 patients undergoing cardiac surgery were enrolled between November 2017 and August 2019, and 316 patients were excluded for the following reasons: 280 due to surgery types, 11 due to severe lung infection, 15 due to severe pulmonary hypertension, 6 due to ejection fraction $(E F)<40 \%$ and 4 due to refusal to sign informed consent. Ninety-five patients were randomized to the mRIPC group ( $\mathrm{m}=$ 47 ) or to the control group $(n=48)$. During the follow-up, some patients were further excluded due to perioperative death, secondary surgery and the inability to contact patients. Forty-three patients in each group were included in the analysis (Fig. 1).

The baseline demographic and clinical characteristics are shown in Table 1. The mRIPC group had a higher rate of spontaneous return to cardiac rhythm $(79.1 \%$ vs. $60.5 \%, P=0.033)$ and a lower rate of reperfusion arrhythmia $(16.3 \%$ vs. $32.6 \%, P=0.044)$. In addition, there were no significant differences between the other baseline variables before and during surgery. The results of postoperative outcomes are presented in Table 2. The duration of postoperative mechanical ventilation was significantly decreased in the mRIPC group (18.57 \pm 3.27 vs. $20.22 \pm 3.76, P=0.033)$. 
Table 1

Baseline and clinical characteristics of the patients

\begin{tabular}{|c|c|c|c|}
\hline Characteristic & $\operatorname{mRIPC}(n=43)$ & Control $(n=43)$ & $P$ value \\
\hline Age (years) & $53.3 \pm 8.8$ & $54.7 \pm 10.0$ & 0.487 \\
\hline Sex (male/female) & $18 / 25$ & $20 / 23$ & 0.157 \\
\hline Weight (kg) & $62.6 \pm 9.0$ & $63.7 \pm 11.7$ & 0.662 \\
\hline LVEF (\%) & $56.76 \pm 9.73$ & $58.48 \pm 9.16$ & 0.171 \\
\hline $\mathrm{FEV}_{1} / \mathrm{FVC}(\%)$ & $81.50 \pm 9.58$ & $84.69 \pm 9.18$ & 0.334 \\
\hline Euro SCORE II (score) & $3.1 \pm 1.4$ & $3.0 \pm 1.5$ & 0.714 \\
\hline Hypertension (n, \%) & $25(58.1 \%)$ & $23(53.5 \%)$ & 0.157 \\
\hline Diabetes mellitus $(\mathrm{n}, \%)$ & $12(27.9 \%)$ & $11(25.6 \%)$ & 0.187 \\
\hline Atrial fibrillation $(n, \%)$ & $27(62.8 \%)$ & $30(69.8 \%)$ & 0.143 \\
\hline Unbearable numbness and pain ${ }^{\#}(n, \%)$ & $0(0 \%)$ & $0(0 \%)$ & \\
\hline Crystalloid infusion (ml) & $1229 \pm 265$ & $1302 \pm 289$ & 0.224 \\
\hline Colloid infusion (ml) & $373 \pm 139$ & $387 \pm 157$ & 0.648 \\
\hline Urine (ml) & $896 \pm 253$ & $845 \pm 233$ & 0.338 \\
\hline \multicolumn{4}{|l|}{ Type of procedure } \\
\hline $\operatorname{MVR}(n, \%)$ & $14(32.6 \%)$ & $16(37.2 \%)$ & 0.162 \\
\hline MVR + TVP (n, \%) & $29(67.4 \%)$ & $27(62.8 \%)$ & 0.162 \\
\hline \multicolumn{4}{|l|}{ Valve type } \\
\hline Mechanic (n, \%) & $42(97.7 \%)$ & $43(100 \%)$ & 0.500 \\
\hline Tissue $(n, \%)$ & $1(2.3 \%)$ & $0(0 \%)$ & 0.500 \\
\hline Aortic cross-clamping time (min) & $52.28 \pm 7.51$ & $54.88 \pm 6.34$ & 0.086 \\
\hline Bypass time (min) & $75.07 \pm 17.36$ & $78.49 \pm 23.19$ & 0.450 \\
\hline Surgery time $(\mathrm{h})$ & $3.26 \pm 0.56$ & $3.37 \pm 0.68$ & 0.448 \\
\hline \multicolumn{4}{|c|}{ The data are presented as the mean \pm SD or number $(\%)$. } \\
\hline \multicolumn{4}{|l|}{${ }^{*}$ Compared with the control group, $P<0.05$. } \\
\hline \multicolumn{4}{|c|}{ \#Patients experienced intolerable numbness or pain during mRIPC intervention. } \\
\hline \multicolumn{4}{|c|}{$\begin{array}{l}\text { LVEF, left ventricular ejection fraction; MVP, mitral valve replacement; TVP, tricuspid valvuloplasty; } \\
\text { Euro SCORE, European System for Cardiac Operative Risk Evaluation; ICU, intensive care unit. }\end{array}$} \\
\hline
\end{tabular}




\begin{tabular}{|llll|}
\hline Characteristic & mRIPC $(\mathbf{n = 4 3 )}$ & Control $(\mathbf{n = 4 3 )}$ & P value \\
\hline spontaneous return to cardiac rhythm $(\mathrm{n}, \%)$ & $34(79.1 \%)^{*}$ & $26(60.5 \%)$ & 0.033 \\
\hline Reperfusion arrhythmia $(\mathrm{n}, \%)$ & $7(16.3 \%) *$ & $14(32.6 \%)$ & 0.044 \\
\hline The data are presented as the mean \pm SD or number $(\%)$. & & \\
\hline${ }^{\star}$ Compared with the control group, $P<0.05$. & & \\
\hline \#Patients experienced intolerable numbness or pain during mRIPC intervention. & \\
\hline $\begin{array}{l}\text { LVEF, left ventricular ejection fraction; MVP, mitral valve replacement; TVP, tricuspid valvuloplasty; } \\
\text { Euro SCORE, European System for Cardiac Operative Risk Evaluation; ICU, intensive care unit. }\end{array}$ \\
\hline
\end{tabular}

Table 2

Postoperative outcomes of the patients

\begin{tabular}{|llll|}
\hline Characteristic & mRIPC $(\mathbf{n}=\mathbf{4 3})$ & Control $(\mathbf{n}=\mathbf{4 3})$ & $P$ value \\
\hline ICU ventilation time $(\mathrm{h})$ & $18.57 \pm 3.27$ & $20.22 \pm 3.76$ & 0.033 \\
\hline ICU stay $(\mathrm{h})$ & $39.04 \pm 11.12$ & $41.62 \pm 8.37$ & 0.23 \\
\hline Postoperative hospital stays $(\mathrm{d})$ & $13.07 \pm 2.45$ & $14.02 \pm 4.17$ & 0.201 \\
\hline Postoperative ALI $(\mathrm{n}, \%)$ & $8(18.6 \%)$ & $15(34.9 \%)$ & 0.046 \\
\hline The data are presented as the mean \pm SD or number $(\%)$. & \\
\hline *Compared with the control group, $P<0.05$. & & \\
\hline ICU = intensive care unit. & & \\
\hline
\end{tabular}

There was no significant difference in cTnl, CK-MB or LDH concentration between the two groups at T0. Compared to the control, modified RIPC significantly decreased the serum concentrations of cTnl, CK-MB and LDH at T2, T3 and T4 $(P<0.01)$, and the IS was also decreased $(P<0.01)$. In addition, the concentration of CK-MB at T1 was also lower than that in the control group (Fig. 3).

Compared to the control group, the $\mathrm{PaO}_{2} / \mathrm{FiO}_{2}$ of the intervention group was higher at $\mathrm{T} 4(362.02 \pm$ $103.53 \mathrm{~mm} \mathrm{Hg}$ vs. $309.41 \pm 105.00 \mathrm{~mm} \mathrm{Hg}, P=0.022$; Fig. $4 \mathrm{~A}$ ), and the incidence of ALI was decreased (18.60\% vs. $34.88 \%, P=0.046$; Table 2 ).

There were no significant differences in IL-6, IL-10 or TNF-a concentrations between the two groups at T0. At T1, T2, T3, and T4, mRIPC significantly decreased the level of IL-6 $(P<0.01$; Fig. 5A). Compared with patients in the control group, patients in the mRIPC group had higher levels of IL-10 at T1 and T4 $(P<$ 0.05 ; Fig. 5C), with the increase in IL-10 being more obvious at T3 and T4 $(P<0.01$; Fig. 5C), and lower levels of TNF-a at T1 and T4 $(P<0.05$; Fig. 5B), with the decrease in TNF-a being more obvious at T3 and T4 $(P<0.01$; Fig. 5B). In addition, the difference in Lac between the two groups at T2, T3, and T4 was also statistically significant $(P<0.05$; Fig. 5D). 


\section{Discussion}

In this clinical study, we demonstrate for the first time that modified RIPC induced three times can reduce the levels of myocardial injury-associated markers at multiple time points in patients undergoing MVR surgery, inhibit the inflammatory response, enhance oxygenation function and decrease the incidence of ALI.

Remote ischemic preconditioning (RIPC), as a noninvasive organ protection method initially proposed by Pryzklenk [12], has been shown to help remote organs withstand a subsequent prolonged ischemic event and provide multiorgan protection [13]. Previous preclinical studies $[14,15]$ and some clinical studies [16, 17] have shown that RIPC had a good protective effect on myocardial injury during cardiac surgery. The study results of Thielmann and colleagues [5] showed that RIPC provided perioperative myocardial protection and improved the prognosis of patients undergoing elective CABG surgery. However, some studies did not obtain positive results $[18,19]$. These findings are in line with the report of the 2 largescale prospective trials on RIPC in cardiac surgery $[6,7]$. These contradictory results make the organ protection effects of RIPC controversial. Some researchers speculate that most of the existing studies were focused on patients undergoing CABG surgery; these patients often have transient myocardial ischemia before the operation, which may induce a protective effect similar to that of RIPC [20]. In this study, we selected patients who underwent elective MVR, and most of these patients had no history of myocardial ischemia before surgery. In addition, we also used a modified RIPC method, which was carried out at three time points before surgery, so that the organ protection from the early and late time windows of RIPC can be overlapped. Our results demonstrated that mRIPC could significantly decrease the release of myocardial enzymes (cTnl, CK-MB and LDH) at multiple time points (T2, T3 and T4) after surgery, and the IS was also decreased $(P<0.01)$. Myocardial enzymes represented by $\mathrm{cTn}$ I have been proven to be sensitive in the evaluation of myocardial injury, and the IS is also a good index to measure the use of vasoactive drugs after surgery [21]. In addition, modified RIPC intervention has been confirmed to have higher rate of spontaneous return fo cardiac rhythm and reduce the incidence of reperfusion arrhythmia, which also shows its protective effect on the heart. Previous studies have shown that a single RIPC stimulation can reduce myocardial enzyme release and provide myocardial protection after valve surgery $[22,23]$, which is similar to our results. However, a recent study involving valve surgery and CABG did not support the protective effect of a single RIPC [24]. The combined use of propofol and volatile anesthetic regimens during surgery may be one of the reasons for this finding, and preoperative complications such as diabetes mellitus may also affect the results of the study. Previous an experimental animal study has confirmed that the inherent protective effect of RIPC might be fully exploited by a volatile anesthetic itself [25]. In contrast, propofol has been considered by some studies to reverse the protective effect of RIPC $[26,27]$. To observe the protective effect of RIPC more effectively, propofol was used to maintain anesthesia in this study.

To date, no study has addressed the optimal site and duration of the RIPC stimulus or the optimal number of repetitions. The study of Kim and colleagues [28] demonstrated that delayed RIPC (24-48 $\mathrm{h}$ before surgery) did not reduce myocardial enzyme release after CABG. It is worth mentioning that a study 
recently published by Meersch et al [29] showed that high-dose RIPC could stimulate increases in tissue inhibitor of metalloproteinases-2 and insulin-like growth factor-binding protein 7 increases in patients refractory to low-dose RIPC and decrease the incidence of AKI after cardiac surgery. Both of them are methods to enhance the effect of RIPC. Our research provides a new direction for exploring optimal RIPC modalities in the future. Three complete preoperative interventions may cause pain or numbness, but our study showed that no patient had moderate or severe unbearable pain.

Our results showed that mRIPC was able to improve oxygenation and decrease the incidence of ALI after mitral valve replacement surgery. The RCT study by Li et al. [30] reported that limb RIPC reduced the incidence of ALI by improving pulmonary oxygenation in patients without severe pulmonary disease after lung resection under propofol-remifentanil anesthesia. Another study found that limb RIPC may improve gas exchange after lobectomy by reducing the levels of EBC 8-isoprostane and other markers of oxidative lung injury [31]. Similar results were obtained from a recently published meta-analysis [32]. This metaanalysis included 10 clinical studies of cardiovascular surgery, and the final analysis showed that the RIPC group could reduce the expression of TNF-a, shorten the duration of ICU mechanical ventilation, and improve postoperative pulmonary function in patients undergoing cardiac surgery. Although the effect of RIPC on the incidence of major cardiovascular and cerebrovascular events after cardiac surgery is controversial, recent studies and our results show that RIPC has a positive protective effect on postoperative pulmonary function in patients undergoing cardiac surgery or noncardiac surgery. It is noted that although there was statistical difference in oxygenation index at T4 after surgery, a $\mathrm{PaO}_{2} / \mathrm{FiO}_{2}$ difference of $50 \mathrm{~mm} \mathrm{Hg}$ in the $300 \mathrm{~mm} \mathrm{Hg}$ range are not clinically important differences. However, the incidence of postoperative ALI was significantly decreased, and further research is needed to explore the protective effect of mRIPC on lung after cardiac surgery.

Regarding the mechanism of RIPC organ protection, it is believed that ischemic stimulation can induce multiple pathways to play a protective role in target organs, which is achieved by regulating the nerve reflex and secretion of humoral factors [33]; adenosine, IL-6, TNF-a, IL-10 and other humoral factors have been confirmed to regulate the role of RIPC. Our study showed that mRIPC was able to reduce the expression of the proinflammatory cytokines IL- 6 and TNF- $\alpha$ and increase the expression of the antiinflammatory factor IL-10. In addition, compared with the control group, the serum Lac level was decreased in the mRIPC group, which was consistent with the findings of previous studies [34]. It is suggested that the ischemic inflammatory response induced by RIPC and the change in inflammatory mediators may be one of the vital mechanisms of RIPC in perioperative myocardial protection and lung protection, and more studies are needed to explore its mechanism in the future.

Our study has certain limitations. First, our study is not a double-blind design, which may affect the quality of the study. The main reason is that the research design did not include a sham RIPC group. Second, as a single center, small sample clinical study, and only the changes of some biochemical indexes such as myocardial enzymes were statistically significant, but some important clinical outcomes were not recorded in this study, the promotion of study conclusions may be limited. In addition, the RIPC stimulation mode in this study was three cycles, but some studies used four cycles, and the optimal 
stimulation mode of RIPC is still controversial. Last, this study only focused on the short-term clinical outcomes during the perioperative period, without longer-term follow-up. The main outcome of myocardial protection was the change in myocardial enzymes, and less attention was paid to the major cardiovascular clinical events. In this study, there were no significant differences in ICU stay, length of stay or mortality during hospitalization between the two groups. We are preparing to design and implement a larger clinical study with a longer follow-up time to obtain more clinical data.

\section{Conclusions}

Modified remote ischemic preconditioning can provide myocardial and lung protective effects in patients undergoing mitral valve replacement surgery and can be performed safely and easily in clinical applications. Further studies are needed to investigate the protective effects of modified RIPC on longterm clinical outcomes and other organs other than the heart and lung.

\section{Abbreviations}

RIPC: Remote ischemic preconditioning; mRIPC: Modified remote ischemic preconditioning; MVR : Mitral valve replacement; cTnl: Cardiac troponin I; CK-MB: Creatine kinase isoenzyme; LDH: Lactate dehydrogenase; IS: Inotropic score; ALI: Acute lung injury; CPB: Cardiopulmonary bypass; I/R: Ischemiareperfusion; CABG: Coronary artery bypass grafting; EF: Ejection fraction; COPD: Chronic obstructive pulmonary disease; ABP: Arterial blood pressure; CVP: Central venous pressure; TEE: Transesophageal echocardiography; TCI: Target-controlled infusion; ACT: Active clotting time; ICU: Intensive care unit; A$\mathrm{aDO}_{2}$ : Alveolar-arterial oxygen gradient; LOS: Length of stay. IL-6: Interleukin-6; IL-10: Interleukin-6; TNF-a: Tumor necrosis factor-a; Lac: Lactic acid; ELISA: Enzyme-linked immunosorbent assay.

\section{Declarations}

\section{Ethics approval and consent to participate}

The study was approved by the ethics committee of the Affiliated Hospital of Xuzhou Medical University, Xuzhou, Jiangsu Province, China (XYFY2016-KL035-01). It was performed with the 1964 Helsinki Declaration and its later amendments or comparable ethical standards. All participants provided written informed consent .

\section{Consent for publication}

Not applicable.

\section{Availability of data and material}

The datasets generated and/or analyzed during the current study are available from the corresponding author on reasonable request since the database of the research center is not open to the public. 


\section{Competing interests}

The authors report no conflicts of interest in this work.

\section{Funding}

This study was supported by the Research Project of the Health Department of Xuzhou, Jiangsu Province (KC17199).

\section{Authors' contributions}

ZLQ contributed to study design, interpretation of data, and drafted the manuscript. ZK contributed to study design, and interpretation of the data,

drafted the manuscript. GTC was primarily responsible for the processing and analysis of blood samples. XJJ contributed to analysis. SMZ was responsible for the follow up. ZJ contributed to approve the final version. LJD contributed to study design, interpretation of data, and approved the manuscript. All authors have read and approved the final manuscript.

\section{Acknowledgements}

Thanks to Jiangsu Province Key Laboratory of Anesthesiology for its guidance in blood sample analysis.

\section{Authors' Information}

${ }^{1}$ Department of Anesthesiology, The Second Affiliated Hospital of Soochow University, Soochow, Jiangsu 215008, China. ${ }^{2}$ Department of Anesthesiology, The Affiliated Hospital of Xuzhou Medical University, Xuzhou, Jiangsu 221004, China.

${ }^{3}$ Jiangsu Province Key Laboratory of Anesthesiology, Xuzhou Medical University, Xuzhou, Jiangsu 221004, China.

\section{References}

[1]. Roth GA, Johnson C, Abajobir A, Abd-Allah F, Abera SF, Abyu G, et al. Global, Regional, and National Burden of Cardiovascular Diseases for 10 Causes, 1990 to 2015. Journal of the American College of Cardiology. 2017;70(1):1-25.

[2]. Ferdinandy P, Hausenloy DJ, Heusch G, Baxter GF, Schulz R, Levy FO. Interaction of Risk Factors, Comorbidities, and Comedications with Ischemia/Reperfusion Injury and Cardioprotection by Preconditioning, Postconditioning, and Remote Conditioning. Pharmacological Reviews. 2014;66(4):1142-74. 
[3]. Cho YJ, Kim WH. Perioperative Cardioprotection by Remote Ischemic Conditioning. Int J Mol Sci 2019;20:1-22.

[4]. Sivaraman V, Pickard JMJ, Hausenloy DJ. Remote ischaemic conditioning: cardiac protection from afar. Anaesthesia. 2015;70(6):732-48.

[5]. Thielmann M, Kottenberg E, Kleinbongard P, Wendt D, Gedik N, Pasa S, et al. Cardioprotective and prognostic effects of remote ischaemic preconditioning in patients undergoing coronary artery bypass surgery: a single-centre randomised, double-blind, controlled trial. The Lancet. 2013;382(9892):597-604.

[6]. Meybohm P, Bein B, Brosteanu O, Cremer J, Gruenewald M, Stoppe C, et al. A Multicenter Trial of Remote Ischemic Preconditioning for Heart Surgery. New England Journal of Medicine. 2015;373(15):1397-407.

[7]. Hausenloy DJ, Candilio L, Evans R, Ariti C, Jenkins DP, Kolvekar S, et al. Remote Ischemic Preconditioning and Outcomes of Cardiac Surgery. New England Journal of Medicine. 2015;373(15):1408-17.

[8]. Basalay M, Barsukevich V, Mastitskaya S, Mrochek A, Pernow J, Sjöquist P-0, et al. Remote ischaemic pre- and delayed postconditioning - similar degree of cardioprotection but distinct mechanisms. Experimental Physiology. 2012;97(8):908-17.

[9]. Hausenloy DJ, Yellon DM. The Second Window of Preconditioning (SWOP) Where Are We Now? Cardiovascular Drugs and Therapy. 2010;24(3):235-54.

[10]. Zarbock A, Schmidt C, Van Aken H, Wempe C, Martens S, Zahn PK, et al. Effect of Remote Ischemic Preconditioning on Kidney Injury Among High-Risk Patients Undergoing Cardiac Surgery. Jama. 2015;313(21):2133.

[11]. Fathil MFM, Md Arshad MK, Gopinath SCB, Hashim U, Adzhri R, Ayub RM, et al. Diagnostics on acute myocardial infarction: Cardiac troponin biomarkers. Biosensors and Bioelectronics. 2015;70:209-20.

[12]. Przyklenk K, Bauer B, Ovize M, Kloner RA, Whittaker P. Regional Ischemic 'Preconditioning' Protects Remote Virgin Myocardium From Subsequent Sustained Coronary Occlusion. Circulation. 1993;87(3):8939.

[13]. Candilio L, Hausenloy D. Is there a role for ischaemic conditioning in cardiac surgery? F1000Research. 2017;6:563.

[14]. Hausenloy DJ, Yellon DM. Remote ischaemic preconditioning: underlying mechanisms and clinical application. Cardiovascular Research. 2008;79(3):377-86.

[15]. Loukogeorgakis SP, Williams R, Panagiotidou AT, Kolvekar SK, Donald A, Cole TJ, et al. Transient Limb Ischemia Induces Remote Preconditioning and Remote Postconditioning in Humans by a 
KATPChannel-Dependent Mechanism. Circulation. 2007;116(12):1386-95.

[16]. Candilio L, Malik A, Ariti C, Barnard M, Salvo CD, Lawrence D, et al. Effect of remote ischaemic preconditioning on clinical outcomes in patients undergoing cardiac bypass surgery: a randomised controlled clinical trial. Coronary artery disease. 2014;0:1-8.

[17]. Ali ZA, Callaghan CJ, Lim E, Ali AA, Reza Nouraei SA, Akthar AM, et al. Remote Ischemic Preconditioning Reduces Myocardial and Renal Injury After Elective Abdominal Aortic Aneurysm Repair: A Randomized Controlled Trial. Circulation. 2007;116(11_suppl):I-98-I-105.

[18]. Hausenloy DJ, Yellon DM. "Conditional Conditioning" in cardiac bypass surgery. Basic Research in Cardiology. 2012;107(3).

[19]. Rahman IA, Mascaro JG, Steeds RP, Frenneaux MP, Nightingale P, Gosling P, et al. Remote Ischemic Preconditioning in Human Coronary Artery Bypass Surgery: From Promise to Disappointment? Circulation. 2010;122(11_suppl_1):S53-S9.

[20]. Donato M, Evelson P, Gelpi RJ. Protecting the heart from ischemia/reperfusion injury. Current Opinion in Cardiology. 2017;32(6):784-90.

[21]. Yang L, Wang G, Du Y, Ji B, Zheng Z. Remote Ischemic Preconditioning Reduces Cardiac Troponin I Release in Cardiac Surgery: A Meta-Analysis. Journal of Cardiothoracic and Vascular Anesthesia. 2014;28(3):682-9.

[22]. Wu Q, Gui P, Wu J, Ding D, Purusram G, Dong N, et al. Effect of Limb Ischemic Preconditioning on Myocardial Injury in Patients Undergoing Mitral Valve Replacement Surgery. Circulation Journal. 2011;75(8):1885-9.

[23]. Xie JJ, Liao XL, Chen WG, Huang DD, Chang FJ, Chen W, et al. Remote ischaemic preconditioning reduces myocardial injury in patients undergoing heart valve surgery: randomised controlled trial. Heart. 2012;98(5):384-8.

[24]. Moscarelli M, Fiorentino F, Suleiman MS, Emanueli C, Reeves BC, Punjabi PP, et al. Remote ischaemic preconditioning in isolated aortic valve and coronary artery bypass surgery: a randomized trialt. European Journal of Cardio-Thoracic Surgery. 2019;55(5):905-12.

[25]. Behmenburg F, van Caster P, Bunte S, Brandenburger T, Heinen A, Hollmann MW, et al. Impact of Anesthetic Regimen on Remote Ischemic Preconditioning in the Rat Heart In Vivo. Anesthesia \& Analgesia. 2018;126(4):1377-80.

[26]. Bunte S, Behmenburg F, Eckelskemper F, Mohr F, Stroethoff M, Raupach A, et al. Cardioprotection by Humoral Factors Released After Remote Ischemic Preconditioning Depends on Anesthetic Regimen. Critical Care Medicine. 2019;47(3):e250-e5. 
[27]. Kottenberg E, Thielmann M, Bergmann L, Heine T, Jakob H, Heusch G, et al. Protection by remote ischemic preconditioning during coronary artery bypass graft surgery with isoflurane but not propofol - a clinical trial. Acta Anaesthesiologica Scandinavica. 2012;56(1):30-8.

[28]. Kim TK, Min JJ, Cho YJ, Hausenloy DJ, Ahn H, Kim K-H, et al. Effects of delayed remote ischemic preconditioning on peri-operative myocardial injury in patients undergoing cardiac surgery - $\mathrm{A}$ randomized controlled trial. International Journal of Cardiology. 2017;227:511-5.

[29]. Meersch M, Küllmar M, Pavenstädt H, Rossaint J, Kellum JA, Martens S, et al. Effects of Different Doses of Remote Ischemic Preconditioning on Kidney Damage Among Patients Undergoing Cardiac Surgery: A Single-Center Mechanistic Randomized Controlled Trial. Critical Care Medicine. 2020;48(8):e690-e7.

[30]. Li C, Xu M, Wu Y, Li YS, Huang WQ, Liu KX. Limb Remote Ischemic Preconditioning Attenuates Lung Injury after Pulmonary Resection under Propofol-Remifentanil Anesthesia. Anesthesiology 2014:1-11.

[31]. García-de-la-Asunción J, Bruno L, Perez-Griera J, Galan G, Morcillo A, Wins R, et al. Remote Ischemic Preconditioning Decreases Oxidative Lung Damage After Pulmonary Lobectomy. Anesthesia \& Analgesia. 2017;125(2):499-506.

[32]. Zheng L, Han R, Tao L, Yu Q, Li J, Gao C, et al. Effects of remote ischemic preconditioning on prognosis in patients with lung injury: A meta-analysis. Journal of Clinical Anesthesia. 2020;63:109795.

[33]. Tsibulnikov SY, Maslov LN, Gorbunov AS, Voronkov NS, Boshchenko AA, Popov SV, et al. A Review of Humoral Factors in Remote Preconditioning of the Heart. Journal of Cardiovascular Pharmacology and Therapeutics. 2019;24(5):403-21.

[34]. Wenwu Z, Debing Z, Renwei C, Jian L, Guangxian Y, Pingbo L, et al. Limb Ischemic Preconditioning Reduces Heart and Lung Injury After an Open Heart Operation in Infants. Pediatric Cardiology. 2009;31(1):22-9.

\section{Figures}




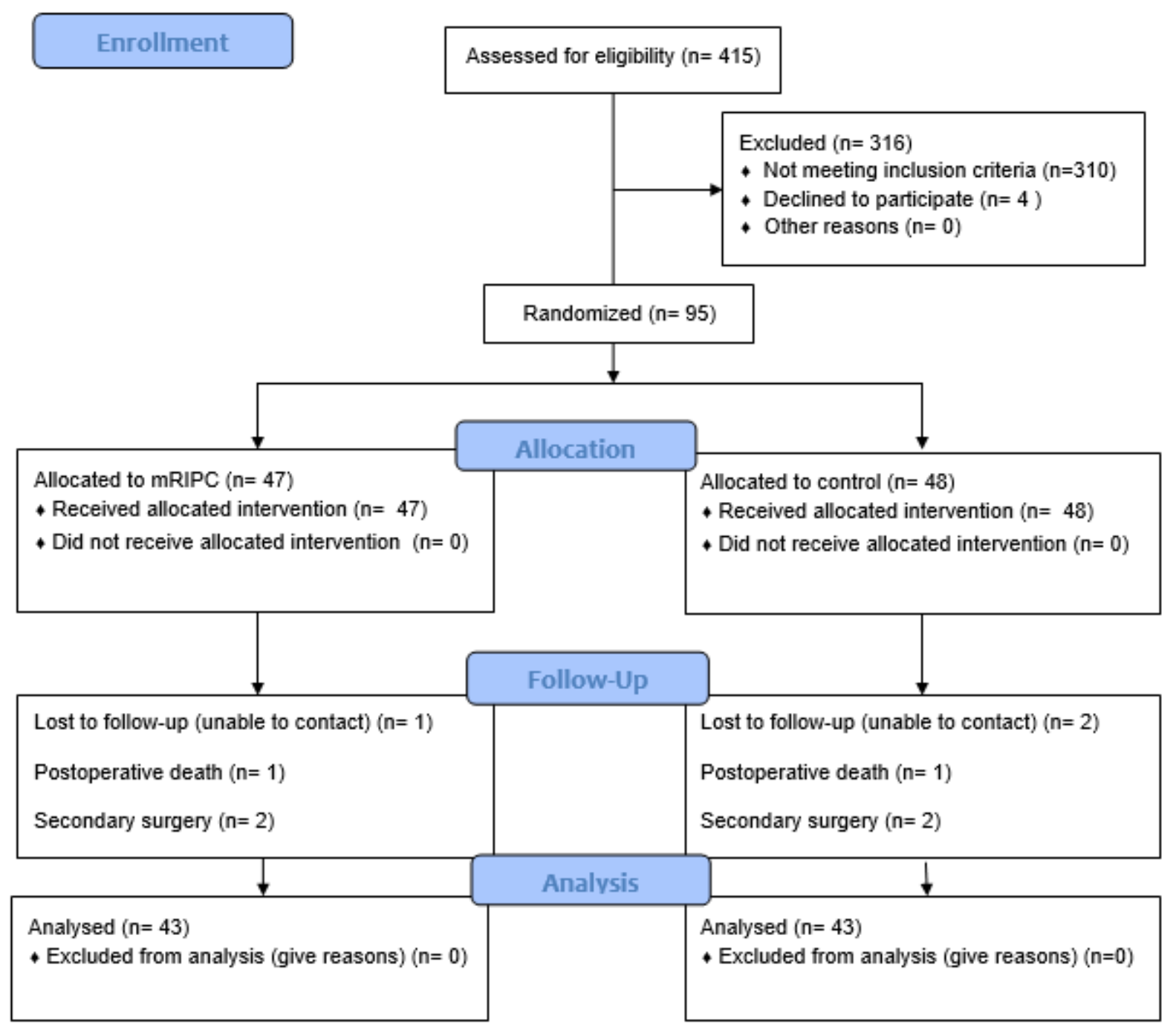

Figure 1

CONSORT flow diagram for the study. 

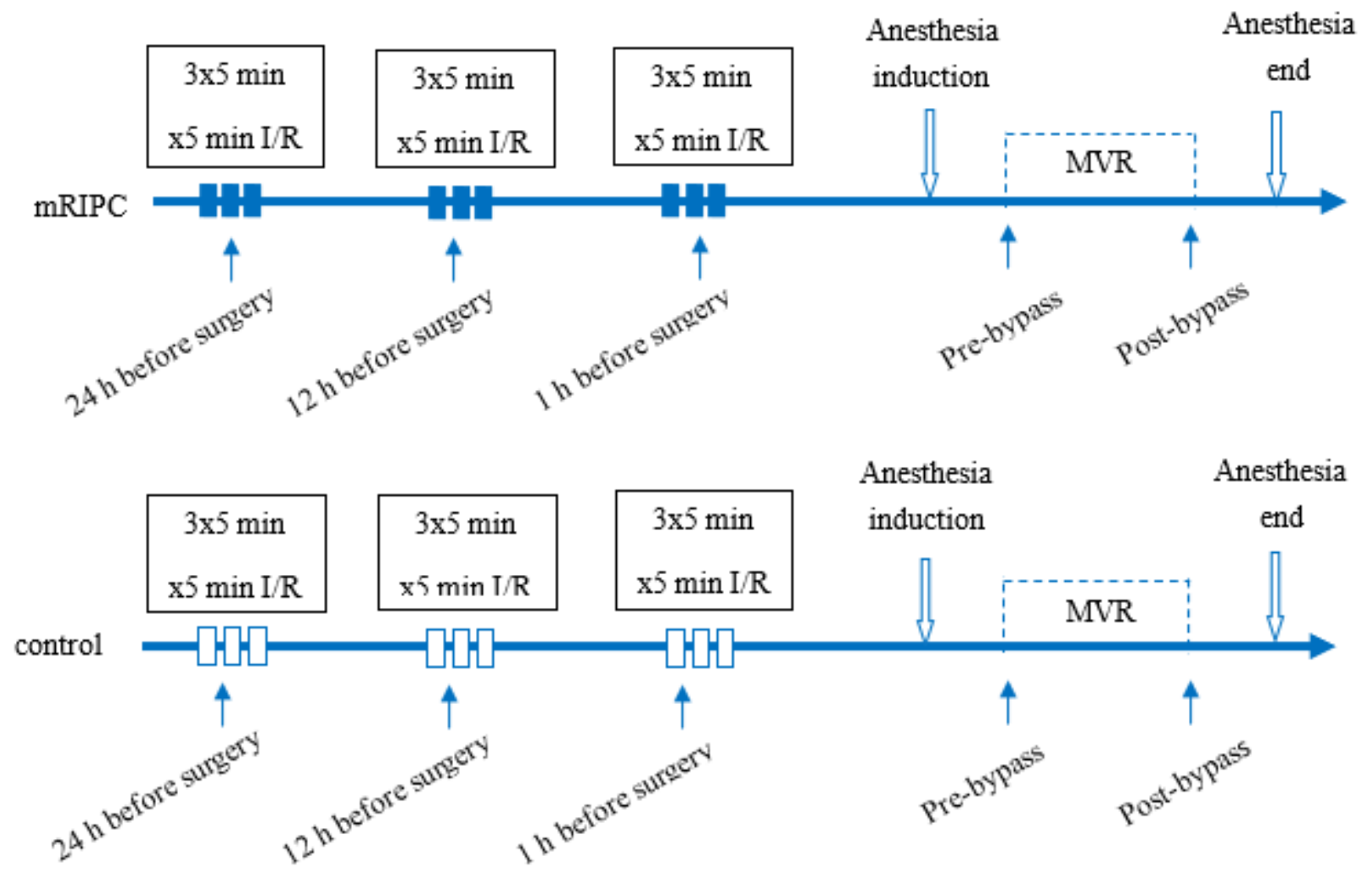

Figure 2

The mRIPC protocol of this study. 

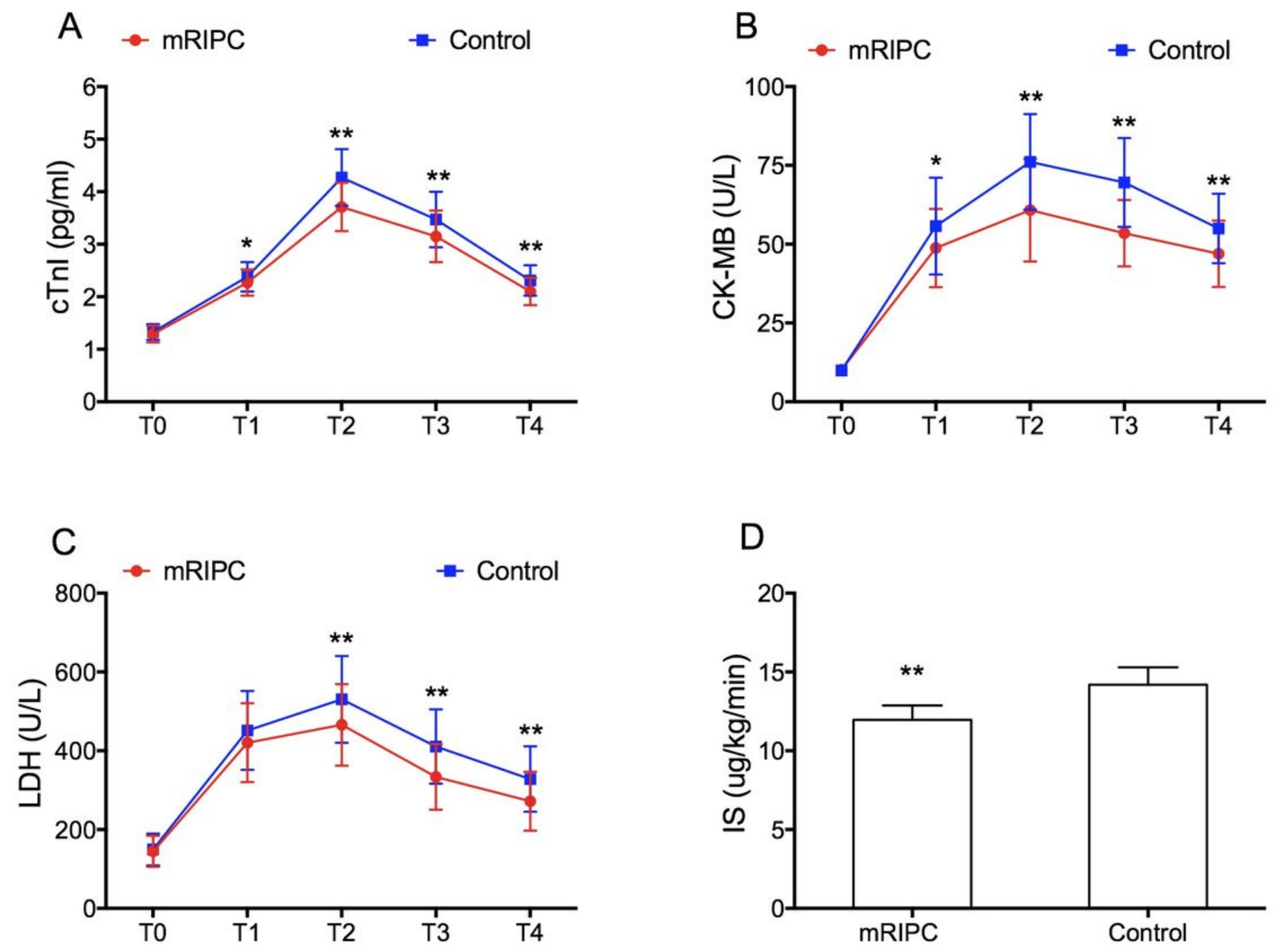

Figure 3

Serum myocardial enzyme content and IS in the two groups. Figure 3 A Serum cTnl content in the two groups. Figure 3B Serum CK-MB content in the two groups. Figure 3C Serum LDH content in the two groups. Figure 3D IS in the two groups. ${ }^{*}$ Compared with the control group, $\mathrm{P}<0.05 .{ }^{*}$ Compared with the control group, $\mathrm{P}<0.01$. cTnl= cardiac troponin $\mathrm{l} ; \mathrm{CK}-\mathrm{MB}=$ creatine kinase isoenzyme; $\mathrm{LDH}=$ lactate dehydrogenase; IS= inotropic score. $T 0=10 \mathrm{~min}$ after intubation, $\mathrm{T} 1=1 \mathrm{~h}$ after aortic declamping, $\mathrm{T} 2=6 \mathrm{~h}$ after surgery, $\mathrm{T} 3=12 \mathrm{~h}$ after surgery, $\mathrm{T} 4=24 \mathrm{~h}$ after surgery. 


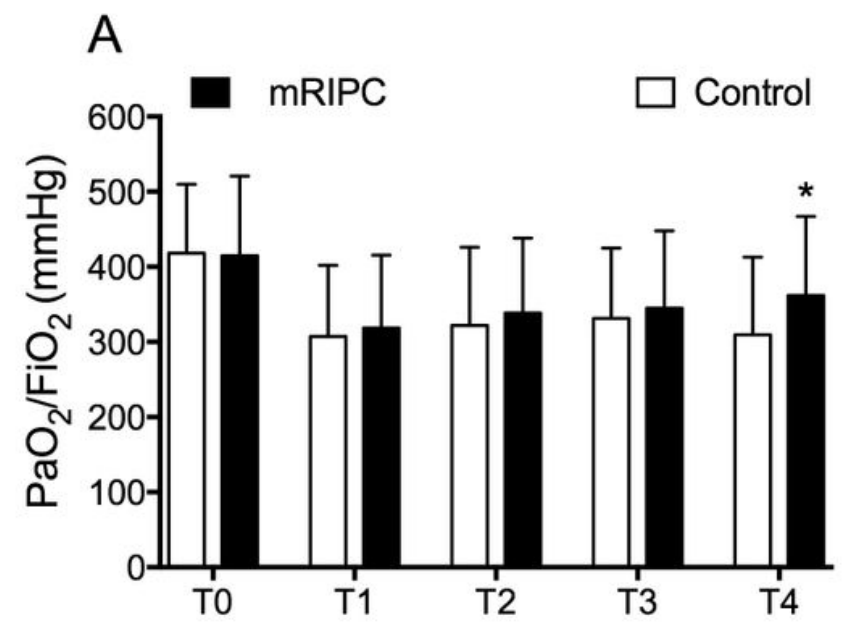

B

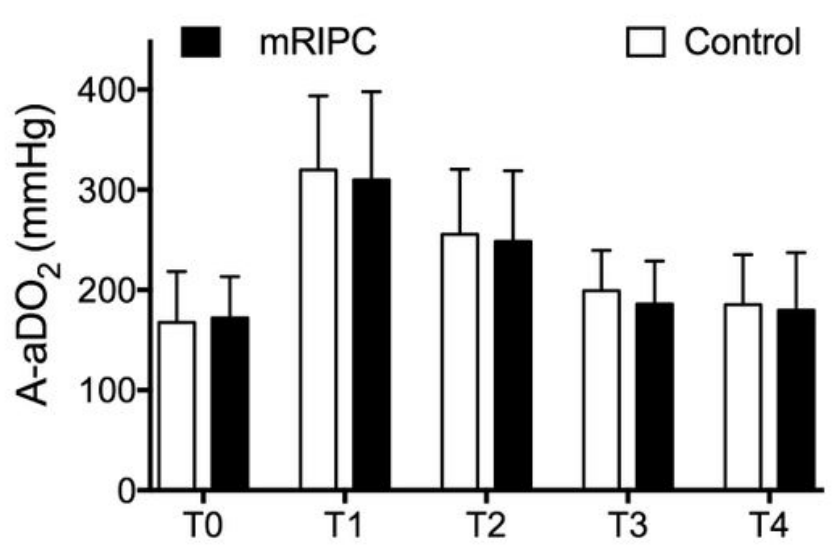

Figure 4

The postoperative outcome of pulmonary oxygenation function in the two groups. Figure 4A PaO2/FiO2 in the two groups. Figure 4B A-aDO2 in the two groups. ${ }^{*}$ Compared with the control group, $\mathrm{P}<0.05 . \mathrm{T} 0=10$ min after intubation, T1 $=1 \mathrm{~h}$ after aortic declamping, T2 $=6 \mathrm{~h}$ after surgery, T3=12 $\mathrm{h}$ after surgery, T4=24 $\mathrm{h}$ after surgery. 

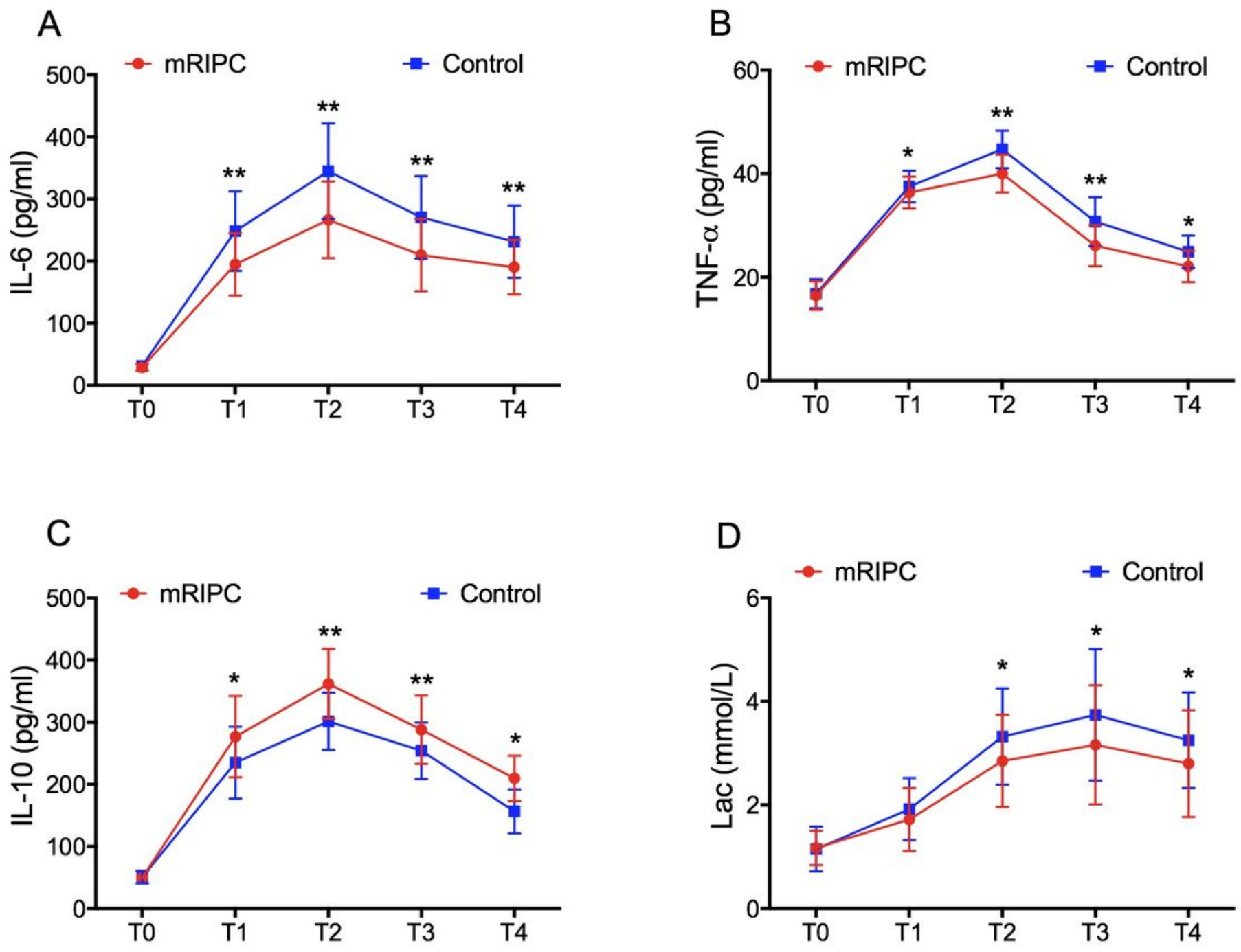

\section{Figure 5}

Comparison of inflammatory mediators and lactic acid values between the two groups at different time points after surgery. Figure 5A IL-6 serum concentration in the two groups. Figure 5B TNF-serum concentration in the two groups. Figure 5C IL-10 serum concentration in the two groups. Figure 5D Lac serum concentration in the two groups. ${ }^{*}$ Compared with the control group, $\mathrm{P}<0.05 .{ }^{* *}$ Compared with the control group, $\mathrm{P}<0.01 . \mathrm{IL}=$ Interleukin; $\mathrm{TNF}=$ tumor necrosis factor; $\mathrm{Lac}=$ lactic acid. $\mathrm{T} 0=10 \mathrm{~min}$ after intubation, $\mathrm{T} 1=1 \mathrm{~h}$ after aortic declamping, $\mathrm{T} 2=6 \mathrm{~h}$ after surgery, $\mathrm{T} 3=12 \mathrm{~h}$ after surgery, $\mathrm{T} 4=24 \mathrm{~h}$ after surgery.

\section{Supplementary Files}

This is a list of supplementary files associated with this preprint. Click to download.

- CONSORTChecklist.doc 